\title{
Extensions of the Stoney formula for substrate curvature to configurations with thin substrates or large deformations
}

\author{
L. B. Freund \\ Division of Engineering, Brown University, Providence, RI 02912 \\ J. A. Floro \\ Sandia National Laboratories, Albuquerque, NM 87185-1415 \\ E. Chason \\ Division of Engineering, Brown University, Providence, RI 02912
}

\begin{abstract}
Two main assumptions which underlie the Stoney formula relating substrate curvature to mismatch strain in a bonded thin film are that the film is very thin compared to the substrate, and the deformations are infinitesimally small. Expressions for the curvature-strain relationship are derived for cases in which these assumptions are relaxed, thereby providing a basis for interpretation of experimental observations for a broader class of film-substrate configurations.
\end{abstract}

PACS: 06.30.B, 68.60.B, 83.10.F 


\title{
DISCLAIMER
}

\begin{abstract}
Portions of this document may be illegible in electronic image products. Images are produced from the best available original document.
\end{abstract}


Curvature based techniques for the measurement of stress in thin films are gaining increasingly widespread use [1-5]. The Stoney formula [6] serves as a cornerstone of experimental work on stress measurement in thin films bonded to substrates. In its most basic form, the formula provides an expression for the curvature $\kappa$, of the substrate in terms of the residual force $f$ in the film (interpreted as a force per unit distance along the interface) due to misfit or other residual elastic strain; this expression is

$$
\kappa_{S t}=\frac{6 f}{h_{s}^{2} M_{s}}
$$

where $h_{s}$ and $M_{s}$ are the thickness and the bi-axial elastic modulus of the substrate. The formula does not involve the properties of the film material, nor does it presume of any particular through-the-thickness distribution of the film stress with resultant $f$.

The formula (1) follows from an analysis of a model of the film-substrate system which is based on several assumptions, and the point of the present discussion is to examine the range of applicability of some of these assumptions in light of current practice $[7,8]$. The main assumptions are: $(i)$ both the film and substrate thicknesses are small compared to the lateral dimensions; (ii) the film thickness is much less than the substrate thickness; (iii) the substrate material is homogeneous, isotropic and linearly elastic, and the film material is isotropic; (iv) edge effects near the periphery of the substrate are inconsequential and all physical quantities are invariant under change in position parallel to the interface; $(v)$ all stress components in the thickness direction vanish throughout the material; $(v i)$ the strains and rotations are infinitesimally small. Current measurement technology is applied in situations where the validity of one or more of these assumptions is questionable. As a guide to the interpretation of data, more exact expressions are derived here for the relationship between mismatch strain and curvature when assumptions $(i i)$ and $(v i)$ are relaxed. The first case treated is that with film and substrate of similar thicknesses, but the deformations are still small. Then, nonlinear deformations are taken into account.

To make the discussion definite, attention will be focused on a system with a circular substrate of radius $R$, the only lateral dimension of consequence. Results are identical for other substrate shapes in the linear deformation regime, and only small quantitative differences arise for nonlinear deformations. For the time being, it will be assumed that the film material is also homogeneous and the stress is uniform through the thickness of the film. A polar section of the system is shown in the inset in Figure 1, with the film and substrate thicknesses labeled as $h_{f}$ and $h_{s}$, respectively, and similarly for the elastic modulus $E$, Poisson ratio $\nu$ and biaxial 
modulus $M=E /(1-\nu)$. Cylindrical $(r, \theta, z)$ coordinates are adopted with the origin on the geometrical midplane of the substrate and the polar axis, or $z$ direction, perpendicular to the interface as shown.

Mismatch strain is a system parameter denoted by $\epsilon_{m}$; in terms of lattice parameters of the film and substrate, say $a_{f}$ and $a_{s}$, respectively, it is defined for an epitaxial system by $\epsilon_{m}=\left(a_{s}-a_{f}\right) / a_{f}$. Mismatch strain can also arise from difference in thermal expansion characteristics or other physical sources.

Due to hypothesis (v) and axial symmetry, the only nonzero stress components in polar coordinates at any material point are $\sigma_{r r}(r, z)$ and $\sigma_{\theta \theta}(r, z)$; the corresponding elastic strain energy density is

$$
U(r, z)=\frac{E}{2\left(1-\nu^{2}\right)}\left[\epsilon_{r r}(r, z)^{2}+\epsilon_{\theta \theta}(r, z)^{2}+2 \nu \epsilon_{r r}(r, z) \epsilon_{\theta \theta}(r, z)\right]
$$

for the appropriate choice of material constants within either the film or substrate. For small deformation, the elastic strains are conveniently expressed in terms of $u(r)$ and $w(r)$, the radial and transverse displacements of points on the substrate midplane, respectively, as

$$
\epsilon_{r r}=u^{\prime}(r)-z w^{\prime \prime}(r)+\epsilon_{m}, \quad \epsilon_{\theta \theta}=u(r) / r-z w^{\prime}(r) / r+\epsilon_{m}
$$

where the prime denotes differentiation with respect to the argument. The mismatch strain is understood to be identically zero in the substrate, so the strain expressions (3) are valid in either material. The strategy adopted here is to select plausible parametric forms for $u(r)$ and $w(r)$, and then to invoke the principle of stationary potential energy to determine optimal values of the parameters involved.

For small deflections, the radial and transverse deformations are un-coupled and a reasonable choice for the midplane displacement is

$$
u(r)=\epsilon_{0} r+\epsilon_{m}, \quad w(r)=\kappa r^{2} / 2
$$

where $\epsilon_{0}$ represents the extensional strain of the substrate midplane and $\kappa$ represents the curvature of this plane. The total potential energy of the system is calculated in terms of $\epsilon_{0}$ and $\kappa$ according to

$$
V\left(\epsilon_{0}, \kappa\right)=2 \pi \int_{0}^{R} \int_{-h_{s} / 2}^{h_{f}+h_{s} / 2} U(r, z) r d r d z
$$

The equilibrium requirement that potential energy must be stationary is enforced with the conditions $\partial V / \partial \epsilon_{0}=0$ and $\partial V / \partial \kappa=0$ from which it follows that [9]

$$
\kappa=\frac{6 \epsilon_{m}}{h_{s}} h m\left[\frac{1+h}{1+h m\left(4+6 h+4 h^{2}\right)+h^{4} m^{2}}\right]
$$


where $h=h_{f} / h_{s}$ and $m=M_{f} / M_{s}$. This is the generalization of the Stoney formula for uniform mismatch strain in the film for arbitrary thickness ratio and arbitrary modulus ratio. The factor outside the square brackets in (6) is $\kappa_{S t}$, and $\kappa \rightarrow \kappa_{S t}$ as $h \rightarrow 0$.

For the case when $m \approx 1$, an expansion of $(6)$ in powers of $h$ yields $\kappa_{i}=\kappa_{S t} /(1+h)^{3}$. This result implies that, if $m=1$ and $h=h_{f} / h_{s}=0.1$, for example, then the error in use of the Stoney of formula is $30 \%$.

To get a clearer picture of the difference between the Stoney expression and the complete expression in (6), consider the range of parameters $h_{f} / h_{s}$ and $M_{f} / M_{s}$ for which

$$
\left|\frac{\kappa}{\kappa_{S t}}-1\right| \leq \frac{1}{10}
$$

where $\kappa$ as given in (6) is presumed. The solid and dashed curves in Figure 1 indicate the locus of points along which the equality in (7) applies, and the region between the curves is the range in the parameter plane for which the use of the Stoney formula for curvature is accurate to within $10 \%$ of the more complete result.

The issue of large deflection is taken up next. Because of their relatively low bending resistance (compared to extensional resistance at comparable strain) and relatively large lateral extent, plate-like solids are easily deformed into a regime where rotations of material line elements are not small (even though strains are still small), necessitating the use of nonlinear kinematics for a proper description of deformation. In the present context, this can be seen by considering the radial extensional strain induced at the substrate midplane due to transverse deflection alone. With reference to Figure 2, an elementary geometrical construction [10] shows this strain to be essentially $d s / d r-1 \approx \frac{1}{2} w^{\prime}(r)^{2}$. An instructive exercise is to compare the magnitude of this quantity to $\epsilon_{0}$ at $r=R$ for the circumstances in which the deformations are assumed to be infinitesimal, that is, for $w^{\prime}(R)=\kappa R$ and $\epsilon_{0}=\frac{1}{6} \kappa h_{s}$. In this case,

$$
w^{\prime}(R)^{2} / 2 \epsilon_{0}=3 \kappa R^{2} / h_{s}
$$

which is not always small in magnitude. For example, for $h_{s}=100 \mu \mathrm{m}, R=1 \mathrm{~cm}$ and radius of curvature of $\kappa^{-1}=10 \mathrm{~m}$, the value is 0.3 , a significant value compared to 1 .

This effect arises because the initially flat substrate cannot deform into a spherical cap shape without stretching or compressing its midplane. The nonlinear coupling between curvature and stretching is incorporated into the model by retaining the term $\frac{1}{2} w^{\prime}(r)^{2}$ in the expression for radial strain in (3),

$$
\epsilon_{r r}(r, z)=u^{\prime}(r)-z w^{\prime \prime}(r)+\frac{1}{2} w^{\prime}(r)^{2}+\epsilon_{m}
$$


It should be noted that the strains are still small and Hooke's law is still appropriate for a description of material behavior. The main point is that the second-order contribution of rotation to strain can be as significant as the first-order linear effect. (In terms of the nonlinear strain tensor, commonly known as the Lagrange strain in material coordinates, the second-order contributions due to stretching are ignored but the second-order contributions due to rotation are retained.)

If it is assumed that the curvature is uniform for this case as well, then the midplane displacement is taken to be

$$
u(r)=\epsilon_{0} r+\epsilon_{1} r^{3}+\epsilon_{m}, \quad w(r)=\kappa r^{2} / 2
$$

The term in $u(r)$ which is cubic in $r$ contributes to $\epsilon_{r r}$ in the same way as does $w^{\prime}(r)^{2}$, and these two terms compete in minimizing the total potential energy. Also, once the nonlinear description is adopted, the lateral dimension $R$ necessarily enters into any expression for curvature.

The requirement that the potential energy must be stationary under variations in $\kappa, \epsilon_{0}$, and $\epsilon_{1}$ at equilibrium leads to a nonlinear relationship between curvature and mismatch strain which is too complicated to warrant presentation here in its most general form. However, for the case when $h_{f} \ll h_{s}$, it reduces to the compact form

$$
S=K\left[1+\left(1-\nu_{s}\right) K^{2}\right]
$$

where $S=\frac{3}{2} \epsilon_{m} R^{2} h_{f} M_{f} / h_{s}^{3} M_{s}$ is a normalized mismatch strain and $K=\frac{1}{4} R^{2} \kappa / h_{s}$ is a normalized curvature.

A graph of $K$ versus $S$ as given in (11) is shown in Figure 3, where it is compared to the linear curvature-strain relationship based on the same assumptions but ignoring nonlinear kinematics. The most important observation is that nonlinear effects arise for normalized mismatch strain in excess of roughly 0.3 . For $R / h_{s}=100, h_{f} / h_{s}=0.01$ and $M_{f} / M_{s}=1$, this corresponds to a mismatch strain of only $\epsilon_{m} \approx 0.002$. As a practical matter, if nonlinear deformation effects are to be avoided for a given level of $\epsilon_{m}$, then the sample dimensions should be chosen so that the dimensionless ratio $S$ is below about 0.3 or 0.4 .

The assumption of uniform curvature incorporated into (10) is quite extreme in the nonlinear range. To illustrate its limitations, a full nonlinear finite element simulation has been carried out for a configuration with $R / h_{s}=50, h_{f} / h_{s}=0.01$ and $M_{f} / M_{s}=1$. No a priori assumption concerning the deformation is incorporated in the calculation. For $S \leq 0.3$ the 
substrate curvature is indeed uniform and the relationship between $K$ and $S$ is linear. As $S$ is increased beyond this value, however, the curvature becomes increasingly nonuniform along any radius of the substrate midplane. This is illustrated in Figure 3 by means of the dashed curves which show the relationship between the normalized mismatch strain $S$ and the local normalized mean curvature (average of radial curvature and circumferential curvature) at radial distances $r / R=0,1 / 4,1 / 2,3 / 4$ and 1 from the center of the substrate. This shows that the substrate becomes relatively flat near its center and more highly curved near its periphery with increasing mismatch. Values of $S>2$ seem to be beyond the practical range for wafer curvature. However, if the calculations are continued into the range of larger $S$, it is found that the curvature near $r / R=0$ approaches a limiting value with increasing $S$. For very large $S$, the mechanical state tends to become nearly uniform over the inner part of the substrate with a boundary layer behavior near the outer edge, reminiscent of the post-buckling behavior of circular plates [11]. In general, curvature measurements in the nonlinear range require much more careful interpretation to extract information than do measurements in the linear range.

An issue which is noted here, but which will not be discussed further, is that the equilibrium state established for large deformation renders the total potential energy stationary but not necessarily minimum. Indeed, if the possibility of a non-axisymmetric deformation is admitted in the assumed parametric deformation field, the axially symmetric configuration may be unstable if the mismatch strain is large enough. In that case, the system tends toward an asymmetric stable configuration with an ellipsoidal shape instead of the spherical shape $[10,12,13]$.

The research support at Brown University of the Office of Naval Research, Contract N00014-95-1-0239 and the MRSEC Program of the National Science Foundation, Award DMR9632524 is gratefully acknowledged.

Sandia is a multiprogram laboratory operated by Sandia Corporation, a Lockheed Martin Company, for the United States Department of Energy under Contract DE-AC04-94AL85000. 


\section{References}

1. P. A. Flinn, in Thin Films: Stresses and Mechanical Properties, edited by J. C. Bravman, W. D. Nix, D. M. Barnett and D. A. Smith (Mater. Res. Soc. Proc. 130, Pittsburgh, PA, 1989), pp. 41-51.

2. W. D. Nix, Metall. Trans. 20A, 2217 (1989).

3. J. A. Floro, E. Chason, S. R. Lee, R. D. Twesten, R. Q. Hwang and I. B. Freund, J. Elec. Mater. 26, 983 (1997).

4. J. A. Floro, G. A. Lucadamo, E. Chason, L. B. Freund, M. Sinclair, R. D. Twesten and R. Q. Hwang, Phys. Rev. Lett. 80, 4717 (1998).

5. J. A. Floro, E. Chason, M. Sinclair, L. B. Freund and G. A. Lucadamo, Appl.Phys. Lett. $\mathrm{xx}$, to appear (1998).

6. G. G. Stoney, Proc. Roy. Soc. (London) A82, 172 (1909).

7. L. B. Freund, J. Mech. Phys. Solids 44, 723 (1996).

8. M. Finot and S. Suresh, J. Mech. Phys. Solids 44, 683 (1996).

9. L. B. Freund, J. Crystal Growth 132, 341 (1993).

10. L. B. Freund (1997), in Thin Films: Stresses and Mechanical Properties VI, edited by W. W. 'Terberich, H. Gao, J. E. Sundgren and S. P. Baker, (Mater. Res. Soc. Proc. 436, Pittsburgh, PA, 1997), pp. 393-404.

11. K. O. Friedrichs and J. J. Stoker, J. Appl. Mech. 9, A-7 (1942).

12. C. B. Masters and N. J. Salamon, Int. J. Engrg. Sci. 31,915 (1993).

13. M. W. Hyer, J. Compos. Mater. 16, 318 (1982). 


\section{Figure captions}

1. The solid (dashed) curve is the locus of points in the parameter plane for which the Stoney formula overestimates (underestimates) the curvature by $10 \%$. A schematic of the filmsubstrate system showing relevant dimensions appears as an inset.

2. Diagram illustrating the extensional strain which arises from rotation of the substrate midplane, represented approximately by $w^{\prime}(r)$.

3. Normalized curvature $K$ versus normalized mismatch strain $S$ for linear and nonlinear deformation (solid curves), based on assumption of uniform curvature. The dashed curves show the local mean curvature versus mismatch strain at the substrate center $r / R=0$, at the periphery $r / R=1$, and at three intermediate values of radius, based on a complete numerical simulation without assumptions on deformed shape. 


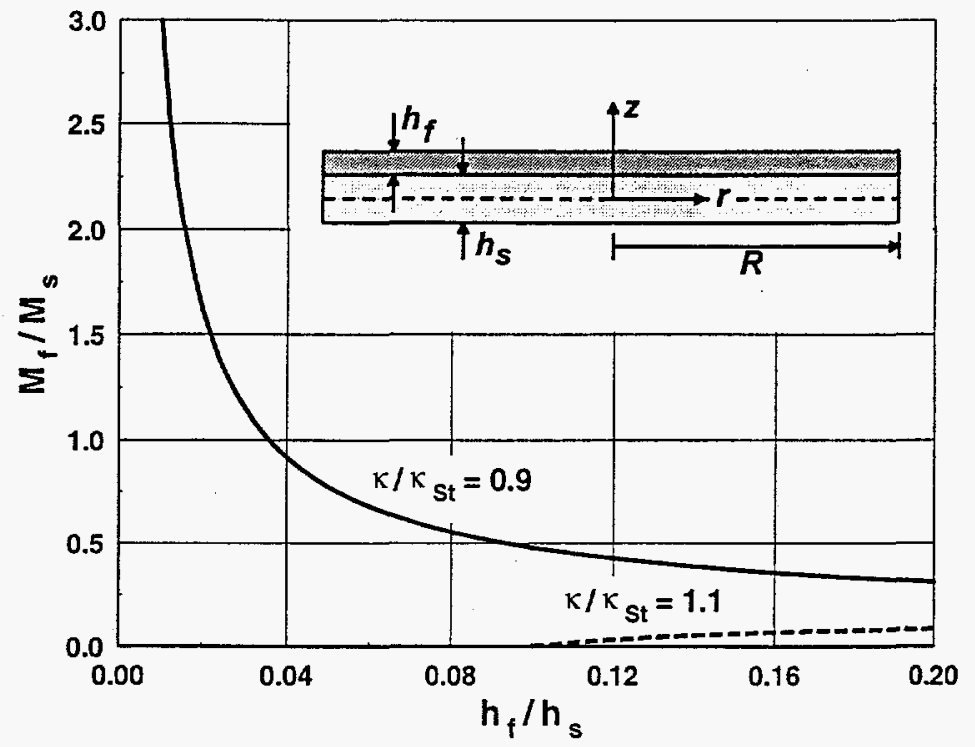

Figure 1: The solid (dashed) curve is the locus of points in the parameter plane for which the Stoney formula overestimates (underestimates) the curvature by $10 \%$. A schematic of the film-substrate system showing relevant dimensions appears as an inset.

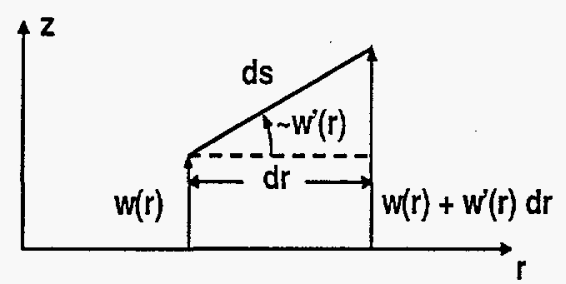

Figure 2: Diagram illustrating the extensional strain which arises from rotation of the substrate midplane, represented approximately by $w^{\prime}(r)$. 


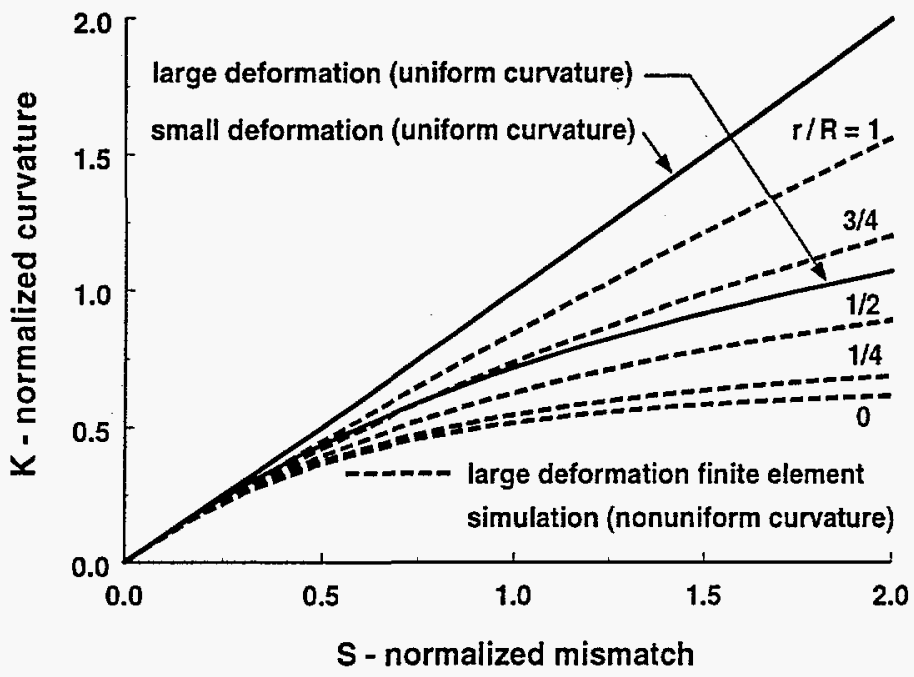

Figure 3: Normalized curvature $K$ versus normalized mismatch strain $S$ for linear and nonlinear deformation (solid curves), based on assumption of uniform curvature. The dashed curves show the local mean curvature versus mismatch strain at the substrate center $r / R=0$, at the periphery $r / R=1$, and at three intermediate values of radius, based on a complete numerical simulation without assumptions on deformed shape. 